His most outstanding contributions to the advance of mathematical knowledge have been in the theory of the Riemann zeta-function and the theory of numbers. The achievement of which, it is believed, he himself is most justly proud is the invention of the 'circle method'. This is a technique of much beauty and generality, which brings great refinement of mathematical analysis to bear on a wide class of unsolved problems in the theory of numbers. The method has been elaborated and improved by other mathematicians, but on its account alone the name of Hardy must for all time rank high among the masters of his subject.

No appreciation of the services of Hardy to the advance of mathematics would be complete which did not attempt to assess the value of his personal influence. Throughout his career he has been the driving force behind a vigorous group of younger research workers. A very considerable proportion of the pure mathematical research now being published in Great Britain is traceable more or less directly to his interest and encouragement, or to the inspiration of his earlier work. His unstinted service during many years to the detailed work of the London Mathematical Society, and the freedom with which his experience and advice are available to all, have established him in a unique position in the regard of British mathematicians.

\section{Hughes Medal}

The Hughes Medal has been awarded to Prof. Arthur Holly Compton.

Prof. Compton has made a number of important contributions to physical science in the field of $\mathrm{X}$-rays and elsewhere. Of late years he has been one of the leaders in the study of cosmic rays.

The experiments of Young and Fresnel early in the nineteenth century proved that light certainly had undulatory properties. But in the present century facts have been emerging, notably in con- nexion with photo-electric action, which are impossible to reconcile with the assumption that light can be described only as an electromagnetic wave of the classical type. These difficulties disappeared if light of frequency $v$ is assumed to be dynamically equivalent to a collection of particles of energy $h \nu(h$ is Planck's constant).

It occurred to Compton that from this point of view the interaction between radiation and free electrons is very simple, and in fact is the simplest interaction which radiation can undergo. Associated with the energy $h v$, according to the electromagnetic theory, there is momentum $h v / c$ ( $c$ is velocity of light). The interaction is thus reduced to a very ancient problem, that of the encounter of two infinitesimal billiard balls with known energies and moments. As the radiation moves with the velocity of light, in most cases the electron can be treated as if it were at rest. It is then obvious that in the collision the electron will acquire energy from the radiation, and the conservation of momentum requires that if the electron moves off in a certain direction the radiation will travel in a certain other direction. But reduction of energy of a quantum of radiation means increase in wave-length, and this increase will be a predetermined function of the direction of the 'scattered' radiation and of the direction of motion of the 'recoil' electron.

Compton published these conclusions in 1922. In 1923 he established the change in wave-length, first qualitatively by Barkla's absorption coefficient methods and then quantitatively with the X-ray spectrometer. In the succeeding years he investigated the energies of the recoil electrons as a function of their direction of motion and showed that the correlation, predicted by the theory, between the direction and energy of the recoil electrons on one hand and the direction and ehange of wave-length of the radiation on the other did in fact occur. This correlation is of fundamental importance in the general theory of the interaction of radiation with matter.

\title{
BIOLOGICAL APPLICATIONS OF SYNTHETIC CHEMISTRY
}

$\mathrm{P}^{\mathrm{n}}$ ROF. J. W. COOK, formerly at the Royal Cancer Hospital, who recently succeeded the late Prof. George Barger as regius professor of chemistry in the University of Glasgow, opened the winter session of the evening meetings in Edinburgh of the Pharmaceutical Society with a lecture on "Some Biological Applications of Synthetic Chemistry".

Prof. Cook said that many new facts have been recorded regarding the natures and the functions of substances which play vital parts in the process of life, and large numbers of these substances have been prepared artificially. Many rare compounds, not known in Nature, and yet possessing powerful biological activity, have arisen from the creative efforts of the synthetic chemist. The spectacular results which followed the introduction of the sulphonamide drugs were the outcome of purely chemical investigations. There is no doubt that prontosil, with its colourless prototype sulphanilamide, is destined to be regarded as one of the greatest boons to mankind conferred by this present age. Of the many thousands of sulphonamides that have now been prepared and examined, none surpasses sulphanilamide in efficacy against streptococcal infections. A later product, M. and B. 693, a sulphapyridine, has robbed pneumonia of much of its terror, and the analogous thiazole derivative has been claimed as effective against infections due to staphylococcus. These claims have not, however, been substantiated by tests carried out in Great Britain.

Much progress has also been made in other branches of chemotherapy; new products are constantly being evolved which have therapeutic and pharmacological properties resembling, and sometimes exceeding, those of natural plant products. Carefully planned researches, such as those of the Drug Addiction Committee of the United States Public Health Service, should receive every possible encouragement. The very rapid decline in the use of cocaine as an addiction drug, after the discovery of synthetic substitutes such as procaine, gave a stimulus to efforts to solve the problem of drug addiction with the opium 
alkaloids, and the efforts of the American investigators have been concentrated on a study of the pharmacology of a wide range of morphine derivatives and of numerous classes of synthetic compounds having structural features in common with some of those of the morphine molecule.

The chemotherapy of tubercular infections, Prof. Cook said, is now receiving increasing attention. Sir Robert Robinson has recently summarized progress made in this field, pointing out that in tuberculosis, as in leprosy, a suitable therapeutic agent should provide a means of penetrating or breaking down the waxy envelope which surrounds the bacilli of these infections. The active component of chaulmoogra oil, long used in the treatment of leprosy, is known to be a cyclopentenyl fatty acid. Many synthetic analogues of chaulmoogric acid also possess leprocoidal activity, and the nature of these substances gives support to the view that they owe their activity to a capacity to effect impairment of function of the fatty envelope of the organisms.

Prof. Cook also reviewed in the course of his lecture the progress made in the past ten years in the chemistry of the vitamins and the members of the sterol class, including several groups of hormones. Most of the known vitamins, he said, have been isolated in a state of chemical purity, and the principal ones have been prepared synthetically; in addition, the biological role of several of them has been partially elucidated. The pure crystalline vitamin $\mathrm{A}$ was isolated for the first time only a few months ago. The other principal fat-soluble vitamin, known as vitamin $\mathrm{D}$, is now available commercially in chemically pure form. Ascorbic acid, or vitamin
$\mathrm{C}$, is now available as a synthetic product. Incidentally, it has been claimed that vitamins $\mathrm{A}$ and $\mathrm{C}$ increase the body's resistance to infection ; this, said Prof. Cook, is now disputed, although it seems likely that vitamin $A$, by maintaining the tone of the mucosa, renders these less liable to attack by pathogenic organisms. The principal members of the vitamin $B$ complex have been synthesized and considerable insight gained into their biological function. Two other vitamins which have yielded their secrets to chemical investigation in recent years are vitamins $\mathrm{E}$ and $\mathrm{K}$. The availability of the pure synthetic vitamins will hasten the solution of problems concerned with their biochemistry and mode of physiological action.

Remarkable progress has also been witnessed in the chemistry of a group of hormones which are related in molecular structure to each other and to cholesterol, which, Prof. Cook said, may be regarded as their biochemical progenitor. Except in the case of the ostrus-producing hormone, total synthesis has nat yet been achieved, but member's of these groups have been converted into common degradation products, and the steroid hormones have all been prepared artificially from cholesterol or other sterols. Deoxycorticosterone, the most active of the lifemaintenance hormones of the adrenal cortex, has been isolated from ox adrenals and is now prepared artificially in considerable amount. It is concerned in the maintenance of the sodium chloride balance in the blood and has been found effective in the treatment of wound shock; for this reason its availability at the present time is of particular importance.

\section{CONTROL OF INFECTION IN WAR WOUNDS}

$\mathrm{T}$ $\mathrm{HE}$ treatment and care of war wounds is essentially a bacteriological problem, as, indeed, experience in the War of 1914-18 made evident. The chief lethal micro-organisms that are found in infected wounds are Streptococcus pyogenes and the group of anærobic bacilli which cause gas gangrene. Researches within recent years into the causation of puerperal fever have shed light upon the origin of pathogenic streptococci in war wounds; in both cases the chief source of infection is regarded as being droplets of secretion that contain these microbes and that are expelled from the throat of those who nurse and treat the patients; the measure of the risk is given in the statement that $2-5$ per cent of adults harbour Streptococcus pyogenes in the throat. Suitable masks should, therefore, be worn by all those who dress war wounds.

The danger from the intrusion of streptococei into wounds is likely to be much lessened by the administration of drugs of the sulphonamide group, although further experience is needed to define clearly the limits of their usefulness. The prophylaxis of gas gangrene is best attained by the early and adequate surgical cleansing of the wound and by the use of specific antitoxins corresponding to the chief types of pathogenic anærobe. The precise role of chemotherapy in combating infections caused by the gasgangrene group of bacteria is still undetermined; recent experiments with laboratory animals indicate that the most favourable results will be obtained from combined sulphapyridine and antitoxin treatment. Tetanus antitoxin, which confers a passive immunity, still retains its place as an invaluable prophylactic agent ; active immunization with tetanus toxoid-a non-toxic modification of tetanus toxinwas adopted last year as a voluntary method of protection for men in the Army and the Royal Air Force.

A small book on the subject, edited by W. H. Ogilvie and modestly styled a war primer ("War Primer on Wound Infection" : its Causes, Prevention and Treatment. By W. H. Ogilvie, Robert Cruickshank, Lawrence P. Garrod, L. E. H. Whitby and G. A. H. Buttle. Pp. 96. (London: The Lancet, 1940.) 2s. $6 d$. net.) is the work of five recognized authorities, each of whom deals with a particular aspect of the treatment of civil and military wounds. The discussion is arranged under the chapter-headings : the problems to be solved; biological aspect ; bacteriology ; antiseptics ; chemotherapy; surgical principles; and surgical procedures. The present state of knowledge has been set forth by the authors in a succinct and wellbalanced manner. The facts they state and the conclusions they have reached deserve to be studied with the greatest care by everyone whose duty it is to assist in treating war wounds. 